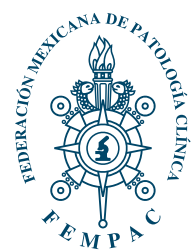

Palabras clave: Diabetes mellitus, tratamiento antidiabético, parámetros bioquímicos, estado redox, peróxidos de lípidos.

Keywords: Diabetes mellitus, diabetes treatment, biochemical parameters, redox state, lipid peroxides.

* Especialista de primer grado (EPG) en Medicina General Integral y Farmacología. Profesora asistente. ‡ EPG en Medicina General Integral y Bioquímica Clínica. Instructor.

$\S$ EPG en Endocrinología. Profesor asistente.

\| Estomatóloga General. EPG en Bioestadística. Profesora Auxiliar. ^EPG en Medicina General Integral e Histología. Profesor Auxiliar.

Universidad de Ciencias Médicas «Carlos J Finlay». Camagüey, Cuba.

Correspondencia: Dra. Mederos-Pérez I E-mail:mpiraida@ infomed.sld.cu

Recibido: 26/06/2019 Aceptado: $25 / 05 / 2020$

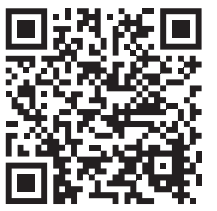

\title{
Efecto del tratamiento farmacológico de la diabetes sobre parámetros bioquímicos clásicos y estado redox
}

\author{
Effect of diabetes's pharmacological treatment over \\ classic biochemical parameters and redox state
}

Mederos-Pérez I,* Vázquez-Silva Y, ${ }^{\ddagger}$ De la Cruz-Fernández CY,\$ López-Lamezón S, " Mederos-Pérez I ${ }^{\mathbb{*}}$

\section{RESUMEN}

Introducción: La diabetes constituye un problema de salud. Los parámetros clínicos y de laboratorio resultan esenciales para evaluar la efectividad del tratamiento y control metabólico. Los fármacos utilizados para su tratamiento mejoran parámetros glucémicos y lipídicos, pero su influencia sobre biomarcadores de estrés oxidativo ha sido menos estudiada. Objetivo: Determinar la influencia del tratamiento farmacológico de la diabetes mellitus tipo 2 sobre parámetros bioquímicos clásicos y estado redox. Material y métodos: Se realizó un estudio analítico transversal en un universo de 29 pacientes que cumplieron los criterios de inclusión y exclusión. Las variables evaluadas fueron: grupo de edad, sexo, tratamiento farmacológico, glucemia, hemoglobina glucosilada, colesterol, HDLc, LDLc, triacilglicéridos y peróxidos de lípidos. Resultados: Predominaron pacientes entre 20 a 49 años con $51.7 \%$ y la monoterapia con $62.1 \%$, siendo la metformina la más empleada $(37.9 \%)$. Los parámetros glucémicos mostraron reducción significativa sólo en los tratados con insulina en monoterapia, al igual que colesterol $(p=0.042), \operatorname{HDLC}(p=0.039)$ y triacilglicéridos $(p=0.043)$; los valores de malondialdehído no mostraron significación en ninguna modalidad de tratamiento. Conclusiones: Metformina fue la terapia más empleada e insulina la variante que mostró significación estadística de las diferencias encontradas en las concentraciones de glúcidos y lípidos.

\section{ABSTRACT}

Introduction: Diabetes is a health problem. Clinical and laboratory parameters are essential to evaluate treatment efficacy and metabolic control. Medication use for treating improve glycemic and lipid parameters, but their influence over oxidative stress biomarkers has been less well-studied. Objective: To determine the influence of type 2 diabetes mellitus treatment over classical biochemical parameters and redox state. Material and methods: A cross-sectional analytical study was conducted among a total of 29 patient who met inclusion-exclusion criteria. The variables under study were: age group, gender, pharmacological treatment, glycaemia, glycosylated hemoglobin, cholesterol, HDLC, LDLc, triglycerides and lipid peroxides. Results: Patients between 20 and 49 years predominated at $51.7 \%$, as well as monotherapy at $62.1 \%$, being metformin the most use medication (37.9\%). Glycemic parameters showed a significant decree only in those patients treated with insulin monotherapy. Cholesterol ( $p=0.042), \operatorname{HDLc}(p=0.049)$ and triglycerides $(p=0.043)$ also decreased; malondialdehyde valves were not significant in any treatment modality. Conclusions: Metformin was the most used treatment while insulin was the one in which the differences found in the concentrations of sugars and lipids were statistically significant.
T a diabetes mellitus tipo 2 (DM2) se describe como un desorden metabólico caracterizado por una hiperglucemia crónica, que tiene como eje central una disfunción de las células beta del páncreas expresada como: una disminución de la respuesta secretora inicial de insulina al incremento de los niveles de glucosa y otros nutrientes en el periodo postprandial, o un aumento de la secreción de insulina en respuesta a la hiperglucemia postprandial mantenida que resulta de la deficiente incorporación de glucosa a la 
célula a consecuencia de la resistencia de los tejidos a la acción de la insulina, o producida simultáneamente por ambos trastornos. ${ }^{1}$

Esta enfermedad constituye un problema de salud que ha venido alcanzando mayor relevancia en el contexto de un aumento constante de su prevalencia y de un mayor impacto en el desarrollo de sus complicaciones. ${ }^{2}$ Si la epidemia no se detiene antes de 25 años, en el mundo habitarán 592 millones de personas con esta enfermedad. ${ }^{2}$

La DM2 es uno de los mayores problemas para los sistemas de salud también en Latinoamérica, región que abarca 21 países y más de 577 millones de habitantes. La Federación Internacional de Diabetes (FID) estimó en el 2011 que la prevalencia ajustada de diabetes en la región era de $9.2 \%$ entre los adultos de 20 a 79 años y un crecimiento en el número de casos para el año 2030 de cerca de 39.9 millones de casos., ${ }^{3,4}$

En Cuba la DM se encuentra dentro de las 10 primeras causas de muerte en todas las edades, con un comportamiento creciente desde el año 2000 hasta la fecha. Al cierre del 2018 se reportan 2,378 defunciones para una tasa de mortalidad bruta de 21.1 por cada 100,000 habitantes, cifra que Camagüey supera con una tasa de 25.0, resultando la cuarta más alta del país. La prevalencia en Cuba es de 64.3 por cada 1,000 habitantes, también superada por esta provincia con valores de $80.4 .^{5}$

La finalidad primordial es detener el aumento de esta enfermedad por las serias consecuencias que representa para la salud. ${ }^{6}$ Las complicaciones crónicas más frecuentes se deben a la micro y macroangiopatía que conduce a complicaciones severas ${ }^{6-9}$ cuya causa metabólica fundamental es la hiperglucemia crónica, capaz de modificar vías metabólicas intracelulares que incrementan el estrés oxidativo y generan importantes cambios estructurales y funcionales a nivel celular. ${ }^{10,11}$

El prolongado periodo preclínico en la DM2 hace que al diagnóstico se muestren ya algunas complicaciones crónicas. El problema merece especial atención, si se añade que aproximadamente entre 30 y $50 \%$ de los pacientes no están diagnosticados a pesar de tener signos clínicos de larga data. ${ }^{12,13}$

El tratamiento de la diabetes se centra en tres objetivos íntimamente relacionados: a) mejorar la utilización celular de la glucosa y otros nutrientes; b) normalizar al máximo posible los niveles de glucemia y otras alteraciones, sin perturbar de manera notable el estilo de vida del paciente, y c) prevenir un buen número de complicaciones graves. ${ }^{14}$

Dada la variedad de opciones terapéuticas disponibles, que incluyen grupos farmacológicos ampliamente estudiados como insulinas, sulfonilureas, biguanidas, tiazolidinedionas, meglitinidas, a los que se suman agonistas del receptor para el péptido similar al glucagón tipo 1 (GLP1, glucagon like peptid 1), inhibidores de la dipeptidil peptidasa-4 e inhibidores del cotransportador sodio-glucosa tipo 1 , con menor tiempo de ingreso al mercado internacional, el tratamiento debe sustentarse en la medicina basada en evidencias e individualizarse según las características del paciente, y será su propia condición la que determine la mejor terapia. ${ }^{14}$

Cuando los tratamientos se inician en etapas relativamente tempranas de evolución de la enfermedad, se reducen al mínimo las complicaciones. Los parámetros clínicos y de laboratorio resultan esenciales para establecer el diagnóstico. Además, permiten efectuar una evaluación integral inicial, valorar la efectividad del tratamiento y forman parte de los criterios de control metabólico. ${ }^{4}$

Cómo influyen los fármacos utilizados para tratamiento de la DM2 en parámetros bioquímicos clásicos está bien sustentado en un sin número de investigaciones; ${ }^{14,15}$ sin embargo, la relación existente entre tratamiento farmacológico y biomarcadores de estrés oxidativo ha sido menos investigada, hecho que motivó la realización de la presente investigación con el objetivo de determinar la influencia del tratamiento farmacológico de la DM2 sobre parámetros bioquímicos que incluyen niveles séricos de peróxidos de lípidos como biomarcador de estrés oxidativo.

\section{MATERIAL Y MÉTODOS}

Se realizó un estudio analítico, transversal, con el objetivo de determinar la influencia del tratamiento farmacológico de la DM2 sobre los parámetros bioquímicos clásicos y estado redox, en pacientes de la provincia Camagüey durante el año 2016. El universo de estudio estuvo constituido por 29 pacientes que acudieron al Centro Provincial de Atención a Pacientes Diabéticos de Camagüey y cumplieron con los siguientes criterios:

Criterios de inclusión: Mayor de 18 años de edad. Haber cumplido con los criterios de la Asociación Latinoamericana de Diabetes (ALAD) para el diagnóstico de DM2. ${ }^{4}$ Haber transcurrido menos de un año luego de diagnosticada la enfermedad.

Criterios de exclusión: Paciente que se encuentre utilizando antioxidantes o que habitualmente consuma acetaminofén. ${ }^{16,17}$

Para la obtención de información se utilizó la historia clínica individual como fuente secundaria y además se tomó muestra de sangre para las determinaciones nece- 
sarias en dos momentos: al ingreso al centro (T0) y a los tres meses de iniciado el tratamiento farmacológico en dicha institución (T1).

La muestra de sangre venosa se tomó después de la primera consulta y se cumplió con los siguientes requisitos: ayuno nocturno de 12 horas y dieta estimada baja en lípidos durante 24 horas antes de la toma de muestra. ${ }^{18}$ Se extrajeron $10 \mathrm{~mL}$ de sangre de la vena mediano-cubital con jeringuilla estéril y se realizaron las siguientes determinaciones:

Glucemia en ayuna: se realizó a través del método glucosa-oxidasa y radioinmunoanálisis ${ }^{19}$ y se tomaron como valores de referencia los siguientes: normal: $4.1 \mathrm{a}$ $6.2 \mathrm{mmol} / \mathrm{L}$; alterada: $>6.2 \mathrm{mmol} / \mathrm{L}$.

Hemoglobina glucosilada (HbA1c): se determinó por el método de interacción antígeno-anticuerpo ${ }^{20}$ y se tomaron como valores de referencia de control para diabéticos, los siguientes: normal: $<7 \%(<53 \mathrm{mmol} /$ mol de $\mathrm{Hb}$ ); alterada: $\geq 7 \%(\geq 53 \mathrm{mmol} / \mathrm{mol} \mathrm{de} \mathrm{Hb})$.

Triacilglicéridos: se determinaron por método enzimático, con el Kit reactivo Monotriglitest20, y se tomaron como valores de referencia para el sexo femenino los siguientes: normal: $0.46-1.60 \mathrm{mmol} / \mathrm{L}$ y alterado: $>1.60$ $\mathrm{mmol} / \mathrm{L}$; y para el sexo masculino normal: 0.68-1.88 $\mathrm{mmol} / \mathrm{L}$ y alterado: $>1.88 \mathrm{mmol} / \mathrm{L}$.

Colesterol: se determinó por método enzimático, con el Kit reactivo Colestest ${ }^{20}$ y se tomaron como valores de referencia los siguientes: normal: 2.9 a $5.2 \mathrm{mmol} / \mathrm{L}$; alterado: > $5.2 \mathrm{mmol} / \mathrm{L}$.

HDLc (cholesterol high-density lipoprotein): para la determinación de HDLc en suero se utilizó el Kit HDL-C enzimático ${ }^{20}$ y se tomaron como valores de referencia los siguientes: normal: $\geq 1.1 \mathrm{mmol} / \mathrm{L}$; alterado: $<1.1 \mathrm{mmol} / \mathrm{L}$.

LDLc (cholesterol low-density lipoprotein): para la determinación de LDLc en suero se utilizó el Kit LDL-C enzimático ${ }^{20}$ y se tomaron como valores de referencia los siguientes: normal: $\leq 2.6 \mathrm{mmol} / \mathrm{L}$; alterado: $>2.6 \mathrm{mmol} / \mathrm{L}$.

Las muestras mencionadas hasta el momento se procesaron en el laboratorio del Hospital Docente Clínico Quirúrgico «Manuel Ascunce Domenech» utilizando analizador automático Hitachi. Se usaron reactivos de producción nacional procedentes del Centro de Productos Biológicos «Carlos J. Finlay» en Ciudad de La Habana, excepto para la hemoglobina glucosilada (HbA1c directa) para la que se utilizó reactivo de producción internacional (Italia: C.P.M.sas).

Se realizó control interno de la calidad utilizando suero control con valores conocidos de concentración de glucosa, triacilglicéridos, colesterol, HDLc y LDLc, en el rango normal y patológico, Elitrol I y II, respectivamente; con un coeficiente de variación $(\mathrm{CV}) \leq 5 \%$.

Peróxidos de lípidos: la determinación de los niveles séricos de peróxidos de lípidos se realizó a través la medición de un compuesto que reaccionan con el ácido tiobarbitúrico, el malondialdehído (MDA), producto aldehído que se forma de la degradación de los peróxidos, presenta un color susceptible de ser medido y tiene la capacidad de reaccionar con el ácido tiobarbitúrico, cuyos valores se expresan en $\mu \mathrm{mol} / \mathrm{L}{ }^{21-23}$ Las muestras se procesaron en el Laboratorio de las Ciencias Básicas Biomédicas de la Universidad de Ciencias Médicas de Camagüey, en un espectrofotómetro US-850.

Para el análisis estadístico de los datos se utilizó el SPSS versión 23.0. En la descripción de las variables se emplearon los estadísticos descriptivos de resumen como frecuencia absoluta, porcentaje y la media aritmética y desviación estándar en variables cuantitativas, así como mínimo y máximo. A través de la prueba no paramétrica de rangos con signos de Wilcoxon se determinó la significación estadística de las diferencias encontradas en las concentraciones de los parámetros bioquímicos medidos durante la aplicación de los diferentes tratamientos. Para ello se utilizó un nivel de significación de 0.05 (5\%).

Los resultados se presentaron en texto, gráficos y tablas estadísticas para su mejor análisis y comprensión.

\section{RESULTADOS}

La distribución de pacientes según edad y sexo (Figura 1), muestra un predominio de casos entre 20 a 49 años con 15 pacientes para $51.7 \%$, con un comportamiento similar en ambos sexos.

Fue utilizado el tratamiento farmacológico en monoterapia en $62.1 \%$ de los pacientes (18) y la terapia combinada en $37.9 \%$ (11). La metformina como terapia única resultó la más empleada con $37.9 \%$ del total de casos, seguido de la insulina con $17.2 \%$. La terapia combinada

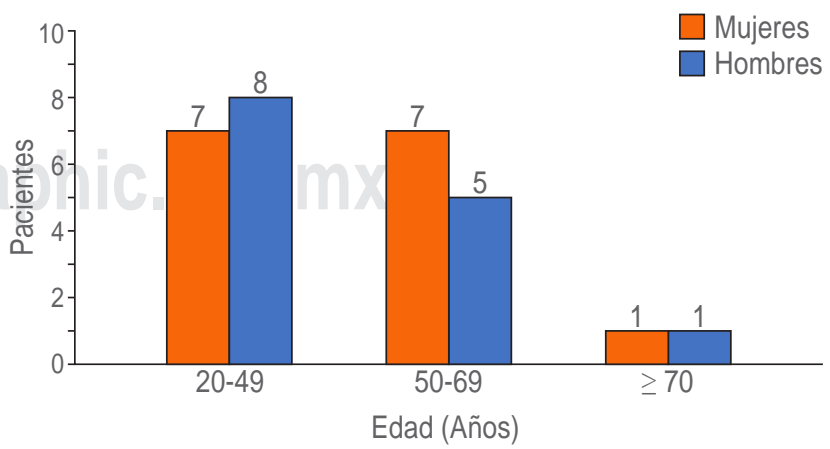

Figura 1: Diabéticos tipo 2 de reciente diagnóstico según edad y sexo. 
más utilizada fue insulina-glibenclamida y metforminaglibenclamida, ambas con $13.8 \%$ (Tabla 1).

Los estadísticos descriptivos y significación estadística de las diferencias encontradas en las concentra-

\begin{tabular}{lcc}
\hline \multicolumn{3}{c}{$\begin{array}{c}\text { Tabla 1: Diabéticos tipo } 2 \text { de reciente diagnóstico } \\
\text { según tratamiento farmacológico (Figura 1). }\end{array}$} \\
\hline Tratamiento & $n$ & $\%$ \\
\hline Insulina & 5 & 17.2 \\
Metformina & 11 & 37.9 \\
Glimepirida & 2 & 6.9 \\
Monoterapia & 18 & 62.1 \\
Insulina-glibenclamida & 4 & 13.8 \\
Insulina-metformina & 3 & 10.3 \\
Metformina-glibenclamida & 4 & 13.8 \\
Terapia combinada & 11 & 37.9 \\
Total & 29 & 100.0 \\
\hline
\end{tabular}

Nota: El porcentaje se calculó a partir del total de pacientes $(\mathrm{N}=29)$. ciones de los parámetros glucémicos según tratamiento farmacológico (Tabla 2) mostraron una reducción de la concentración promedio de estos parámetros en todas las variantes terapéuticas a los tres meses de iniciado el tratamiento, con una desviación estándar (DE) con valores pequeños, lo que se traduce en una poca dispersión de las cifras de los parámetros en estos pacientes. Resultaron significativas $(p=0.043)$ las diferencias encontradas sólo en casos tratados con insulina en monoterapia.

Los parámetros lipídicos según tratamiento farmacológico (Tabla 3) revelan significación estadística de las diferencias encontradas en las concentraciones de colesterol $(p=0.042)$, HDLc $(p=0.039)$ y triacilglicéridos $(p=0.043)$ sólo en los pacientes tratados con insulina en monoterapia.

Los valores de MDA (Tabla 4), representativo de los niveles de peróxidos de lípidos, no mostraron significación en ninguna modalidad de tratamiento, siendo las combinaciones insulina-glibenclamida y metforminaglibenclamida las de mayor aproximación, con valores de $p=0.068$.

Tabla 2: Estadísticos descriptivos y significación estadística de los parámetros glucémicos según tratamiento farmacológico.

\begin{tabular}{|c|c|c|c|c|c|}
\hline Tratamiento & Parámetro & Media $\pm \mathrm{DE}$ & Mínimo & Máximo & Sig. \\
\hline \multirow[t]{4}{*}{ Insulina } & Glicemia 1 & $9.42 \pm 4.16$ & 5.4 & 15.4 & \multirow{2}{*}{$0.043^{*}$} \\
\hline & Glicemia 2 & $6.52 \pm 1.58$ & 5.0 & 9.0 & \\
\hline & $\mathrm{HbA1c} 1$ & $13.7 \pm 10.2$ & 5.2 & 28.7 & \multirow{2}{*}{$0.043^{*}$} \\
\hline & $\mathrm{HbA1c} 2$ & $7.4 \pm 3.0$ & 5.0 & 12.0 & \\
\hline \multirow[t]{4}{*}{ Insulina-glibenclamida } & Glicemia 1 & $9.5 \pm 3.6$ & 6.7 & 14.7 & \multirow{2}{*}{0.068} \\
\hline & Glicemia 2 & $5.9 \pm 1.1$ & 4.6 & 7.0 & \\
\hline & $\mathrm{HbA1c} 1$ & $25.6 \pm 8.6$ & 13.0 & 31.6 & \multirow{2}{*}{0.068} \\
\hline & $\mathrm{HbA1c} 2$ & $9.8 \pm 1.9$ & 7.0 & 11.0 & \\
\hline \multirow[t]{4}{*}{ Insulina-metformina } & Glicemia 1 & $11.4 \pm 2.9$ & 9.1 & 14.6 & \multirow{2}{*}{0.109} \\
\hline & Glicemia 2 & $5.6 \pm 0.7$ & 4.8 & 6.0 & \\
\hline & $\mathrm{HbA1c} 1$ & $11.7 \pm 6.9$ & 7.7 & 19.7 & \multirow{2}{*}{0.109} \\
\hline & $\mathrm{HbA1c} 2$ & $6.6 \pm 0.5$ & 6.0 & 7.0 & \\
\hline \multirow[t]{4}{*}{ Metformina } & Glicemia 1 & $6.3 \pm 2.0$ & 4.6 & 10.0 & \multirow{2}{*}{0.155} \\
\hline & Glicemia 2 & $5.6 \pm 1.1$ & 4.1 & 8.0 & \\
\hline & $\mathrm{HbA1c} 1$ & $6.8 \pm 2.3$ & 2.3 & 11.0 & \multirow{2}{*}{0.285} \\
\hline & $\mathrm{HbA1c} 2$ & $5.3 \pm 1.8$ & 2.6 & 8.0 & \\
\hline \multirow[t]{4}{*}{ Metformina-glibenclamida } & Glicemia 1 & $6.5 \pm 1.8$ & 4.0 & 7.9 & \multirow{2}{*}{0.465} \\
\hline & Glicemia 2 & $8.0 \pm 3.2$ & 4.9 & 12.0 & \\
\hline & $\mathrm{HbA1c} 1$ & $8.5 \pm 2.1$ & 6.0 & 11.1 & \\
\hline & $\mathrm{HbA1c} 2$ & $6.9 \pm 0.9$ & 6.0 & 8.1 & 0.180 \\
\hline \multirow[t]{4}{*}{ Glimepirida } & Glicemia 1 & $7.1 \pm 0.9$ & 6.4 & 7.7 & \multirow[b]{2}{*}{0.180} \\
\hline & Glicemia 2 & $4.5 \pm 0.8$ & 3.9 & 5.0 & \\
\hline & $\mathrm{HbA1c} 1$ & $14.1 \pm 11.2$ & 6.1 & 22.0 & \multirow{2}{*}{0.655} \\
\hline & $\mathrm{HbA} 1 \mathrm{c} 2$ & $9.4 \pm 2.5$ & 7.6 & 11.2 & \\
\hline
\end{tabular}

$\mathrm{DE}=$ desviación estándar. ${ }^{*}$ Estadísticamente significativo. 
Tabla 3: Estadísticos descriptivos y significación estadística de los parámetros lipídicos según tratamiento farmacológico.

\begin{tabular}{|c|c|c|c|c|c|}
\hline Tratamiento & Parámetro & Media $\pm \mathrm{DE}$ & Mínimo & Máximo & Sig. \\
\hline \multirow[t]{8}{*}{ Insulina } & Colesterol 1 & $6.5 \pm 3.6$ & 3.5 & 12.4 & \multirow{2}{*}{$0.042^{*}$} \\
\hline & Colesterol 2 & $5.4 \pm 2.4$ & 3.0 & 8.9 & \\
\hline & HDL 1 & $1.4 \pm 0.3$ & 1.0 & 1.9 & \multirow{2}{*}{$0.039^{*}$} \\
\hline & HDL 2 & $1.8 \pm 0.3$ & 1.5 & 2.2 & \\
\hline & LDL 1 & $3.9 \pm 2.3$ & 1.1 & 6.4 & \multirow{2}{*}{0.068} \\
\hline & LDL 2 & $2.6 \pm 1.3$ & 1.1 & 4.4 & \\
\hline & Triglicéridos 1 & $1.4 \pm 0.5$ & 0.8 & 2.0 & \multirow{2}{*}{$0.043^{*}$} \\
\hline & Triglicéridos 2 & $1.2 \pm 0.4$ & 0.7 & 1.8 & \\
\hline \multirow[t]{8}{*}{ Insulina-glibenclamida } & Colesterol 1 & $6.3 \pm 1.7$ & 4.5 & 8.3 & \multirow[b]{2}{*}{0.068} \\
\hline & Colesterol 2 & $4.8 \pm 1.0$ & 3.6 & 6.1 & \\
\hline & HDL 1 & $1.0 \pm 0.3$ & 0.8 & 1.4 & \multirow{2}{*}{0.066} \\
\hline & HDL 2 & $1.7 \pm 0.2$ & 1.4 & 1.9 & \\
\hline & LDL 1 & $3.7 \pm 1.8$ & 2.2 & 6.2 & \multirow{2}{*}{0.068} \\
\hline & LDL 2 & $2.2 \pm 0.8$ & 1.4 & 3.3 & \\
\hline & Triglicéridos 1 & $1.6 \pm 0.3$ & 1.4 & 2.1 & \multirow{2}{*}{0.068} \\
\hline & Triglicéridos 2 & $1.1 \pm 0.2$ & 0.9 & 1.3 & \\
\hline \multirow[t]{8}{*}{ Insulina-metformina } & Colesterol 1 & $6.7 \pm 1.5$ & 5.0 & 8.0 & \multirow{2}{*}{0.109} \\
\hline & Colesterol 2 & $4.9 \pm 0.5$ & 4.4 & 5.4 & \\
\hline & HDL 1 & $1.6 \pm 0.5$ & 1.1 & 2.0 & \multirow{2}{*}{0.785} \\
\hline & HDL 2 & $1.7 \pm 0.8$ & 0.8 & 2.3 & \\
\hline & LDL 1 & $3.7 \pm 0.7$ & 3.2 & 4.5 & \multirow{2}{*}{1.000} \\
\hline & LDL 2 & $3.8 \pm 1.4$ & 3.0 & 5.5 & \\
\hline & Triglicéridos 1 & $3.5 \pm 2.6$ & 1.9 & 6.5 & \multirow{2}{*}{0.109} \\
\hline & Triglicéridos 2 & $2.4 \pm 1.1$ & 1.6 & 3.6 & \\
\hline \multirow[t]{8}{*}{ Metformina } & Colesterol 1 & $4.8 \pm 1.1$ & 2.9 & 6.6 & \multirow{2}{*}{0.374} \\
\hline & Colesterol 2 & $4.5 \pm 4.6$ & 2.9 & 5.6 & \\
\hline & HDL 1 & $2.3 \pm 2.9$ & 1.0 & 11.0 & \multirow{2}{*}{0.168} \\
\hline & HDL 2 & $1.7 \pm 0.5$ & 0.9 & 3.0 & \\
\hline & LDL 1 & $2.7 \pm 1.3$ & 1.1 & 5.4 & \multirow{2}{*}{0.889} \\
\hline & LDL 2 & $2.7 \pm 0.8$ & 1.1 & 3.8 & \\
\hline & Triglicéridos 1 & $1.6 \pm 0.6$ & 0.3 & 2.7 & \multirow{2}{*}{0.594} \\
\hline & Triglicéridos 2 & $1.5 \pm 0.6$ & 0.8 & 2.5 & \\
\hline \multirow[t]{8}{*}{ Metformina-glibenclamida } & Colesterol 1 & $5.1 \pm 0.5$ & 4.6 & 5.7 & \multirow{2}{*}{0.715} \\
\hline & Colesterol 2 & $5.5 \pm 0.5$ & 4.9 & 6.1 & \\
\hline & HDL 1 & $1.2 \pm 0.2$ & 1.0 & 1.4 & \\
\hline & HDL 2 & $1.5 \pm 0.8$ & 0.5 & 2.2 & 0.465 \\
\hline & LDL 1 & $5.4 \pm 2.1$ & 2.6 & 7.3 & \\
\hline & LDL 2 & $3.8 \pm 1.1$ & 2.2 & 4.6 & 0.068 \\
\hline & Triglicéridos 1 & $1.4 \pm 0.3$ & 1.0 & 1.6 & \\
\hline & Triglicéridos 2 & $1.5 \pm 0.5$ & 1.1 & 2.3 & 0.465 \\
\hline Glimepirida & Colesterol 1 & $5.0 \pm 1.8$ & 3.7 & 6.3 & \\
\hline & Colesterol 2 & $6.3 \pm 0.7$ & 5.8 & 6.8 & 0.180 \\
\hline & HDL 1 & $1.4 \pm 0.3$ & 1.0 & 1.4 & \\
\hline & HDL 2 & $1.6 \pm 0.8$ & 1.0 & 2.2 & 0.317 \\
\hline & LDL 1 & $4.9 \pm 3.3$ & 2.6 & 7.2 & \\
\hline & LDL 2 & $4.7 \pm 1.2$ & 3.9 & 5.6 & 0.655 \\
\hline & Triglicéridos 1 & $1.8 \pm 0.3$ & 1.6 & 2.0 & \\
\hline & Triglicéridos 2 & $1.3 \pm 0.3$ & 1.1 & 1.5 & 0.180 \\
\hline
\end{tabular}

$\mathrm{DE}=$ desviación estándar. ${ }^{*}$ Estadísticamente significativo. 
Tabla 4: Estadísticos descriptivos y significación estadística de los niveles de MDA según tratamiento farmacológico.

\begin{tabular}{|c|c|c|c|c|c|}
\hline Tratamiento & Parámetro & Media \pm DE & Mínimo & Máximo & Sig. \\
\hline Insulina & $\begin{array}{l}\text { MDA } 1 \\
\text { MDA } 2\end{array}$ & $\begin{array}{l}6.6 \pm 2.4 \\
4.2 \pm 2.1\end{array}$ & $\begin{array}{l}4.0 \\
2.2\end{array}$ & $\begin{array}{l}9.1 \\
7.3\end{array}$ & 0.080 \\
\hline Metformina & $\begin{array}{l}\text { MDA } 1 \\
\text { MDA } 2\end{array}$ & $\begin{array}{l}7.3 \pm 3.0 \\
4.2 \pm 2.1\end{array}$ & $\begin{array}{l}3.6 \\
2.2\end{array}$ & $\begin{array}{r}11.6 \\
7.3\end{array}$ & 0.248 \\
\hline Glimepirida & $\begin{array}{l}\text { MDA } 1 \\
\text { MDA } 2\end{array}$ & $\begin{array}{l}7.1 \pm 3.0 \\
6.8 \pm 0.8\end{array}$ & $\begin{array}{l}7.1 \\
6.2\end{array}$ & $\begin{array}{l}3.0 \\
7.3\end{array}$ & 0.655 \\
\hline Insulina-glibenclamida & $\begin{array}{l}\text { MDA } 1 \\
\text { MDA } 2\end{array}$ & $\begin{array}{l}8.0 \pm 2.2 \\
3.8 \pm 0.8\end{array}$ & $\begin{array}{l}5.8 \\
2.8\end{array}$ & $\begin{array}{r}10.7 \\
4.7\end{array}$ & 0.068 \\
\hline Insulina-metformina & $\begin{array}{l}\text { MDA } 1 \\
\text { MDA } 2\end{array}$ & $\begin{array}{l}8.6 \pm 2.9 \\
6.5 \pm 2.6\end{array}$ & $\begin{array}{l}6.3 \\
4.2\end{array}$ & $\begin{array}{c}11.8 \\
9.3\end{array}$ & 0.109 \\
\hline Metformina-glibenclamida & $\begin{array}{l}\text { MDA } 1 \\
\text { MDA } 2\end{array}$ & $\begin{array}{l}7.2 \pm 2.2 \\
4.7 \pm 1.0\end{array}$ & $\begin{array}{l}4.3 \\
3.8\end{array}$ & $\begin{array}{l}8.9 \\
5.9\end{array}$ & 0.068 \\
\hline
\end{tabular}

$\mathrm{DE}=$ desviación estándar.

\section{DISCUSIÓN}

El rango de edad en pacientes diabéticos tipo 2 de reciente diagnóstico, con 51.7\% de casos entre 20-49 años, difiere de los estándares a nivel internacional, si se tienen en cuenta que en el mundo la edad promedio de debut oscila entre 40-60 años. ${ }^{24}$

Herrero Gil, et $\mathrm{al}^{24}$ en investigación realizada en Madrid a mayores de 14 años con DM2, independientemente del tiempo de diagnosticada, muestra una media de edad de 65 años (DE \pm 0.9 ).

Cuba reporta una mayor prevalencia de DM2 a partir de los 60 años. ${ }^{5}$ Sin embargo, cuando hablamos de incidencia, Valdés Ramos, et $\mathrm{al}^{2}{ }^{25}$ en casos con menos de seis meses de diagnosticada la enfermedad, de la provincia Granma, describe una media de 49.2 años $(D E \pm 9.50)$, resultado similar a los de la presente investigación. En contraste, Mora González, et al, ${ }^{26}$ en casos entre uno y cinco años de diagnosticados, predominó el grupo etario de 70 a 79 años, hecho que pudiera estar relacionado con el mayor tiempo de debut de la enfermedad en estos casos.

Con relación al sexo se describe en el mundo un predominio de mujeres con DM2. Sin embargo, investigadores como Herrero Gil, et al, ${ }^{27}$ en estudio realizado en Madrid, reveló igual por ciento para ambos sexos. En la región de Latinoamérica, México señala que, de los casos ingresados en servicios de salud pública con diagnóstico de DM2, 54.9\% eran mujeres. ${ }^{28}$

Cuba en el año 2018 informó mayor prevalencia en el sexo femenino, con una tasa de 75.1 por cada 1,000 mujeres, ${ }^{5}$ lo que coincide con otros estudios consultados de la provincia de Cienfuegos, ${ }^{29}$ Valdés Ramos, et al, ${ }^{25}$ en Granma y Mora González, et al, ${ }^{26}$ en Mayabeque.

Los datos demográficos varían a medida se modifica el número de años de diagnosticada la enfermedad como criterio de inclusión en las diferentes investigaciones; donde se trabaja con la prevalencia se observa un incremento del sexo femenino y la media de edad, hechos que pudieran estar relacionados con dos elementos: a) a medida que aumenta la edad se incrementa el riesgo de DM, en especial para el sexo femenino; b) existe un comportamiento creciente de la supervivencia del paciente diabético gracias a los esfuerzos mancomunado de asociaciones y ministerios de salud defensores de su mejor control.

Los pacientes con DM2 cada vez cuentan con mayor número de opciones terapéuticas, sin embargo, para los médicos representa un reto elegir el esquema más conveniente. ${ }^{30}$ El tratamiento individualizado garantiza mejor pronóstico, teniendo como referencia los diferentes documentos de consenso internacional, regional y nacional.

La Asociación Americana de Diabetes (American Diabetes Association-ADA $)^{13}$ y la Asociación Europea para el Estudio de la Diabetes (European Association for the Study of Diabetes-EASD) ${ }^{31}$ sugieren iniciar tratamiento farmacológico al momento del diagnóstico, simultáneamente con el inicio de las modificaciones en el estilo de vida.

La presente investigación muestra concordancia con lo que establecen las principales guías donde la metformina constituye fármaco de primera línea en esta enfermedad.

La elección de metformina está a razón de su bajo precio, la elevada efectividad, un perfil favorable a la pérdida de peso y mínimos efectos secundarios. ${ }^{5,31}$ En 
casos que esté contraindicada, se puede iniciar con otro antidiabético oral (ADO). Un inhibidor de dipeptidil peptidasa 4 (iDPP-4) es la mejor alternativa reportada porque no aumenta el peso y tiene poco riesgo de hipoglucemia. ${ }^{5}$ Cuba no dispone de esta línea terapéutica ${ }^{32}$ por lo que, con beneficios demostrados, las sulfonilureas (SU) de segunda generación ocuparían este lugar.

La insulina resultó la segunda modalidad de tratamiento más utilizada, hecho que pudiera estar relacionado con el estado de descompensación metabólica propio del debut, circunstancia en la que la insulina constituye la mejor terapia. ${ }^{5}$

Dentro del perfil glucémico, la HbA1c constituye el mejor parámetro para seguimiento y control del paciente con DM2. Este proporciona información sobre el grado de control en los dos a cuatro meses previos. ${ }^{33}$ Además, está demostrado que una disminución de la $\mathrm{HbA} 1 \mathrm{c}$ de $0.9 \%$ reduce los episodios cardiovasculares en torno al 10-15\% y que cifras cercanas al 7\% reduce las complicaciones microangiopáticas; en consecuencia la mayoría de las guías establecen como meta para este parámetro valores cercanos a $7 \%$.

A las biguanidas se les reporta 1-2\% de disminución de los valores de HbA1c. Saenz A, et al, ${ }^{34}$ en estudios comparativo del efecto sobre la HbA1c de diferentes monoterapias, describe superioridad estadísticamente significativa de la metformina sobre los iDPP4, con efecto sobre los lípidos también superior aunque modesto.

Las SU en el mundo han sido desplazadas por otros grupos farmacológicos como los iDDP4, pero no dejan de formar parte de la segunda línea de tratamiento cuando el paciente no tolera, tiene contraindicada la metformina o no alcanzan las metas en un periodo de tres meses. En la presente investigación la glimepirida fue utilizada en monoterapia sólo en $6.9 \%$ de los casos, con una reducción de la media de HbA1c de 5.3 (14.1 a 9.4).

En los pacientes recién diagnosticados con síntomas de descontrol metabólico o glucemias (> 300 mg/dL o 16.7 $\mathrm{mmol} / \mathrm{L}$ ), o HbA1c muy elevada (> 10\%), debe considerarse iniciar tratamiento con insulina. ${ }^{13}$ En la presente investigación $17.2 \%$ de los casos se encontraban utilizando insulina en monoterapia, modalidad de tratamiento que mostró un descenso estadísticamente significativo de los parámatelos glucémicos; a los que se suma $24.1 \%$ que se encontraban utilizando insulina combinada con ADO. ${ }^{15}$

La insulina es fundamental para favorecer la captación, uso y almacenamiento glucosa, lípidos y aminoácidos; estimula la glucogénesis, lipogénesis y síntesis de proteínas, y también inhibe el catabolismo de estos compuestos. ${ }^{15}$ A la insulina se le reportan efectos potentes sobre los parámetros glucémicos con reducción significativa del valor glucémico en ayuna, posprandial y de HbA1c. Sobre los lípidos también ejerce una acción relevante.
La hiperglucemia crónica induce la producción de anión superóxido y otras especies reactivas, y se produce un desbalance oxidativo que compromete el funcionamiento vascular y favorece la aparición de las complicaciones. ${ }^{26}$ La identificación de este desbalance oxidativo se puede realizar a través de la detección de productos finales de la peroxidación lipídica, como indicadores de la oxidación de lípidos, entre otros. ${ }^{28}$

En relación con la asociación de la peroxidación lipídica y el control de la enfermedad, también los resultados son diversos. Algunos autores han reportado una asociación positiva entre malonildialdehído y el control glucémico; ${ }^{26,28}$ en cambio, Benítez y otros no encontraron asociación entre dichas variables. ${ }^{35}$

En el estudio de Gopaul, con 39 pacientes, se reporta que el incremento en la peroxidación lipídica en diabéticos tipo 2 no se relaciona con la hiperglucemia o la hiperlipidemia. ${ }^{36}$

Los niveles de peróxidos de lípidos no mostraron significación estadística con las modalidades de tratamiento farmacológico utilizadas, hecho que pudiera estar relacionado con la naturaleza potencialmente dañina del proceso de formación de estos compuestos, traducido en daño celular que va desde las mutaciones en el ADN hasta daños estructurales y funcionales al reaccionar con proteínas. Sobre dichos daños no está demostrado que estos fármacos puedan ejercer acción.

No se evidencian otros estudios para establecer comparaciones a nivel nacional e internacional en la bibliografía consultada por la autora.

\section{CONCLUSIONES}

Predominaron los diabéticos de reciente diagnóstico con edades entre 20 y 49 años, con igual incidencia para ambos sexos.

La monoterapia, y en particular la utilización de metformina, fueron las modalidades de tratamiento más empleadas.

Se evidenció relación estadísticamente significativa entre monoterapia con insulina y parámetros glucémicos y lipídicos, exceptuando las lipoproteínas de baja densidad.

No se demostró relación estadísticamente significativa entre el tratamiento farmacológico y los niveles de peróxidos de lípidos.

\section{REFERENCIAS}

1. Braunwald E, Fauci AS, Kasper DL, Hauser SL, Longo DL, Jamenson JR. Harrison's principles of internal medicine. 17th Ed. New York: McGraw-Hill; 2012. 
2. Hernández Yero JA. Diabetes mellitus, hacia dónde vamos y cómo enfrentarla en el siglo XXI. Rev Cubana Endocrinol [Internet]. 2013 [citado 7 Nov 2014]; 24 (1): [aprox. 9 p.]. Disponible en: http://scielo.sld.cu/scielo.php?script=sci_arttext\&pid=S156129532013000100001\&lng=es

3. Mora Morales E. Estado actual de la diabetes mellitus en el mundo. Acta Méd Costarric [Internet]. 2014 [citado 6 Oct 2015]; 56 (2): [aprox. 5 p.]. Disponible en: http://www.redalyc.org/ pdf/434/43431275001.pdf

4. Asociación Latinoamericana de Diabetes (ALAD). Guías sobre el diagnóstico, control y tratamiento de la diabetes mellitus tipo 2 con medicina basada en la evidencia. Revista de la ALAD [Internet]. 2016 [citado 17 Dic 2017]: [aprox. 5p.]. Disponible en: http://ftp. cmw.sld.cu/Documentos/GUIAS_ALAD_2016_1.pdf

5. Cuba. Ministerio de Salud Pública. Anuario Estadístico de Salud 2018 [Internet]. La Habana: Dirección Nacional de Registros Médicos y Estadísticas de Salud; 2018 [citado 20 May 2019]: [aprox. 206 p.]. Disponible en: http://files.sld.cu/dne/files/2019/04/ Anuario-Electronico-Espa\%C3\%B1ol-2018-ed-2019.pdf

6. Bermúdez Lacayo J, Aceituno Vidaur N, Álvarez Oviedo G, Giacaman Abudoj L, Silva Cárcamo H, Salgado A. Comorbilidades en los pacientes con diabetes mellitus tipo 2 del Instituto Nacional del Diabético, Abril-Junio 2016, Tegucigalpa, Honduras. Archivos de Medicina [Internet]. 2016 [citado 31 Mar 2017]; 12 (4): [aprox. 5 p.]. Disponible en: http://web.a.ebscohost.com/ehost/ pdfviewer/pdfviewer?vid =11\&sid $=7657$ e2ca-626f-4fc3-bb8d0ee933acbc57\%40sessionmgr4009\&hid $=4206$

7. Acosta Altamirano G, Frías de León MG, Reyes Montes MR, Vargas Hernández V, Suarez Cuenca JA. Radicales libres y mecanismos de daño tisular en la diabetes mellitus. Rev Fac Med UNAM [Internet]. 2011 [citado 6 Oct 2015]; 54 (3): [aprox. 7 p.]. Disponible en: www.medigraphic.com/pdfs/facmed/un-2011/un113f.pdf

8. Carranza K, Veron D, Cercado A, Bautista N, Pozo W, Veron D et al. Cellular and molecular aspects of diabetic nephropathy; the role of VEGF-A. Nefrología [Internet]. 2015 [citado 1 Jun 2016]; 35 (2): [aprox. 9 p.]. Disponible en: http://31c-d7b337b9bc15\%4 Osessionmgr4002\&hid $=4109$

9. Neves D. Advanced glycation end-products: a common pathway in diabetes and age-related erectile dysfunction. Free Radic Res [Internet]. 2013 [citado 2 Feb 2015]; 47 Suppl 1: [aprox. 6 p.]. Disponible en: http://web.a.ebscohost.com/ehost/pdfviewer/ pdfviewer?vid=13\&sid=901 bee81-755d-4978-b183-e4575dad c4bf\%40sessionmgr4003\&hid $=4106$

10. Monnier V, Sun W, Sell D, Fan X, Nemet I, Genuth S. Glucosepane: a poorly understood advanced glycation end-product of growing importance for diabetes and its complications. Clinic Chem Lab Med [Internet]. 2014 [citado 2 Feb 2015]; 52 (1): [aprox. 10 p.]. Disponible en: http://web.a.ebscohost.com/ehost/pdfviewer/ pdfviewer?vid=11\&sid=901 bee81-755d-4978-b183-e4575dad c4bf\%40sessionmgr4003\&hid $=4106$

11. Real JT, Folgado J, Molina M, Martínez S, Peiro M, Ascaso J. Homocisteína plasmática, $L p(a)$ y marcadores de estrés oxidativo en la vasculopatía periférica en pacientes con diabetes tipo 2. Clin Invest Arterioscler [Internet]. 2016 [citado 2 Feb 2015]; 373: [aprox. 11 p.]. Disponible en: https://www.clinicalkey.es/service/content/ pdf/watermarked/1-sz.0-S0214916816300614.pdf?locale=es_ES

12. DeFronzo RA. Insulin resistance, lipotoxicity, type 2 diabetes and atherosclerosis: the missing links. The Claude Bernard Lecture 2009. Diabetologia. 2010; 53: 1270-1287.

13. American Diabetes Association. Standards of medical care in diabetes. Diabetes Care [Internet]. 2019 [citado 12 Dic 2019]; 42 (1): [aprox. 204 p.]. Disponible en: https://doi.org/10.2337/ dc19-SINT0
14. Florez J, Armijo JA, Mediavilla A. Farmacología humana. España: Masson; 2014. pp. 322-335.

15. Brunton LL, Chabner BA, Knollmann BC, eds. Goodman \& Gilman's: las bases farmacológicas de la terapéutica. 13a ed. México: McGraw-Hill; 2018.

16. Mariaca C, Zapata M, Uribe P. Oxidación y antioxidantes: hechos y controversias. Rev Asoc Colomb Dermatol [Internet]. 2016 [citado 31 Mar 2017]; 24 (3): [aprox. 11 p.]. Disponibleen: http://web.a.ebscohost. com/ehost/pdfviewer/pdfviewer?vid=15\&sid=7657e2ca-626f-4fc3bb8d-0ee933acbc57\%40sessionmgr4009\&hid=4206

17. Grosser T, Smyth E, Garret A. Gerald F. Antinflamatorios, antipiréticos y analgésicos; farmacoterapia de la gota. En: Goodman \& Gilman's: Las bases farmacológicas de la terapéutica. 12a ed. México: McGraw-Hill; 2012. pp. 959-1004.

18. Suardíaz Pareras J, Cruz Rodríguez C, Colina Rodríguez A. Laboratorio clínico. La Habana: Ecimed; 2004.

19. Centro de Productos Biológicos "Carlos J. Finlay" [Internet]. La Habana. 2015 [citado 12 Feb 2016]; [aprox. 9 p.]. Disponible en: http://www.biofinlay.sld.cu/

20. Centro para el Control Estatal de la Calidad de los Medicamentos (CECMED). Lista de diagnosticadores con autorización de comercialización en Cuba [Internet]. 2015 [citado 12 Feb 2016]; [aprox. 6 p.]. Disponible en: http://www.cecmed.sld.cu/Docs/ RegSan/RegSanDiag.pdf

21. Céspedes Miranda E, Riverón Forment G, Alonso Rodríguez C, Cabrera Pérez-Sanz E, Suárez Castillo N, Rodríguez Oropesa K. Estrés oxidativo y excreción urinaria de albúmina y en diabéticos tipo 2. Rev Cubana Invest Bioméd [Internet]. 2015 [citado 9 Jun 2016]; 34 (4): [aprox. 9 p.]. Disponible en: http:// scieloprueba.sld.cu/scielo.php?script =sci_arttext\&pid =S086403002015000400005\&lng=es\&nrm =iso

22. Lima de Castro L, de Carvalho Martínez M, Garcés A, Pacheco J, Cunha F, de Melo Cunha L et al. Hypoalbuminemia and oxidative stress in patients on renal hemodialysis program. Nutrición Hospitalaria [Internet]. 2014 [citado 26 Oct 2016]; 30 (4): [aprox. 7 p.]. Disponible en: http://www.anmm.org.mx/GMM/2015/n2/ GMM_151_2015_2_245-251.pdf

23. Gastell Pérez PL, Pérez Alejo JL. Métodos para medir el daño oxidativo. Rev Cub Med Mil [Internet]. 2000 [citado 2 Feb 2015]; 29 (3): [aprox. 3 p.]. Disponible en: http://scieloprueba.sld.cu/scielo.php?script=sci arttext\&pid $=$ S0138-65572000000300007\&lng =es\&nrm =iso

24. Herrero-Gil AM, Pinillos-Robles J, Sabio-Repiso P, MartínMaldonado JL, Garzón-González G, Gil-de Miguel A. Tendencias sobre los parámetros del grado de control de los pacientes con diabetes tipo 2 desde el año 2010 al año 2015. Aten Primaria [Internet]. 2017 [citado 21 Sept 2017]; 4 (2): [aprox. 8 p.]. Disponible en: http://dx.doi.org/10.1016/j.aprim.2017.04.002

25. Valdés Ramos E, Campos Arjona MC. Características clínicas y frecuencia de complicaciones crónicas en personas con diabetes mellitus tipo 2 de diagnóstico reciente. Rev Cubana Med Gen Integr [Internet]. 2013 [citado 21 Sept 2017]; 29 (2): 121-131.

26. Mora GM, López ROS, Montoya SX. Caracterización clínica y riesgo cardiovascular global en pacientes hospitalizados con diabetes mellitus tipo 2. Revista de Ciencias Médicas de la Habana. 2017; 24 (1): 25-36.

27. Herrero Gil A, Pinillos Robles J, Sabio Repisio P, Martín Maldonado JL, Garzón González G. Grado en que se alcanzan los objetivos de control en pacientes con diabetes de tipo 2 en diferentes grupos poblacionales. Semergen [Internet]. 2016 [citado 05 Sep 2017]; 11 (57): [aprox. 7 p.]. Disponible en: https://www.clinicalkey.es/\#!/ content/journal/1-sz.0-S1138359316302179

28. Sistema Nacional de Vigilancia Epidemiológica. Boletín epidemiológico diabetes mellitus tipo 2, primer trimestre 
- 2013 [Internet]. México: Sistema Nacional de Vigilancia Epidemiológica; 2013 [citado 12 Dic 2015]. Disponible en: http:// www.epidemiologia.salud.gob.mx/doctos/infoepid/bol_diabetes/ dmz_bol1_2013.pdf

29. Zerquera Trujillo G, Vicente Sánchez B, Rivas Alpizar E, Costa Cruz M. Caracterización de los pacientes diabéticos tipo 2 ingresados en el Centro de Atención al Diabético de Cienfuegos. Rev Finlay [Internet]. 2016 [citado 14 Dic 2016]; 6 (4). Disponible en: http://scielo.sld.cu/scielo.php?script=sci_arttext\& pid $=$ S222124342016000400005

30. Ramírez Roca L, Palencia Prado J, Castro Martínez M. Revisión de las guías de tratamiento farmacológico de diabetes mellitus tipo 2 y opinión en Centroamérica. Med Int México [Internet]. 2015 [citado 21 Sept 2017]; 31 (6): 733-748.

31. Inzucchi SE, Bergenstal RM, Buse JB, Diamant M, Ferrannini E, Nauck $M$ et al. Management of hyperglycemia in type 2 diabetes, 2015: a patient-centered approach: update to a position statement of the American Diabetes Association and the European Association for the Study of Diabetes. Diabetes Care. 2015; 38 (1): 140-149.

32. Calvo Barbados DM, Delgado Martínez I. Formulario Nacional de Medicamentos. 4a ed. La Habana: Ecimed; 2014.

33. Bonke F, Donnachie E, Schneider A, Mehring M. Association of the average rate of change in $\mathrm{HbA} 1 \mathrm{c}$ with severe adverse events: a longitudinal evaluation of audit data from the Bavarian Disease Management Program for patients with type 2 diabetes mellitus. Diabetologia [Internet]. 2016 [citado 31 Mar 2017]; 59 (2). Disponible en: http://web.a.ebscohost.com/ehost/ pdfviewer/pdfviewer?vid = 7\&sid=7657e2ca-626f-4fc3-bb8d0ee933acbc57\%40sessionmgr4009\&hid =4206

34. Saenz A, Fernandez-Esteban I, Mataix A, Ausejo M, Roque M, Moher D. Metformin monotherapy for type 2 diabetes mellitus. Cochrane Database Syst Rev. 2005; (3): CD002966.

35. González RM, Perich P, Arranz C. Heterogeneidad de los trastornos metabólicos de las etapas iniciales de la diabetes mellitus 2. Rev Cub. End. 2009; 20 (1). Disponible en: http://scielo.sld.cu/scielo. php?script=sci_arttext\&pid=S156129532009000100003\&lng=es
36. The Look AHEAD Research Group. Long-term effects of a lifestyle intervention on weight and cardiovascular risk factors in individuals with type 2 diabetes mellitus. four-year results of the look AHEAD trial. Arch Intern Med. 2010; 170: 1566-1575.

37. WMA. Declaración de Helsinki de la AMM - Principios éticos para las investigaciones médicas en seres humanos [Internet]. 2013 [citado 01 Sep 2019]: [aprox. 5 p.]. Disponible en: http://www. wma.net/es/30publications/10policies/b3/

\section{ASPECTOS ÉTICOS}

La investigación se realizó conforme a los principios éticos para las investigaciones biomédicas en seres humanos establecidas en la declaración de Helsinki ${ }^{37}$ y en sus posteriores revisiones, siguiendo las recomendaciones del Consejo Científico de la Facultad de Medicina de la Universidad de Ciencias Médicas «Carlos J Finlay» y del Comité de Ética e Investigaciones de dicha facultad. Se respetó la confidencialidad de los documentos revisados, así como la identidad de las pacientes. Se solicitó consentimiento informado para participar en el estudio y se explicó el carácter voluntario de declarar aquellos aspectos que no dañen su dignidad. La autonomía se consideró desde la decisión individual de participar o no en la investigación. Del mismo modo, se sostuvo una interacción justa y benéfica con los pacientes, que incluyó las acciones de educación para la salud, la promoción de estilos de vida saludables y la protección específica; siguiendo de esta forma los principios de respeto por la autonomía del paciente, beneficencia, no maleficencia y justicia. 